\title{
JORDAN DERIVATIONS ON BLOCK UPPER TRIANGULAR MATRIX ALGEBRAS
}

\author{
HOGER GHAHRAMANI
}

Abstract. We provide that any Jordan derivation from the block upper triangular matrix algebra $\mathscr{T}=\mathscr{T}\left(n_{1}, n_{2}, \cdots, n_{k}\right) \subseteq M_{n}(\mathscr{C})$ into a 2 -torsion free unital $\mathscr{T}$-bimodule is the sum of a derivation and an antiderivation.

Mathematics subject classification (2010): 16W25, 47B47, 16S50, 15B99.

Keywords and phrases: Jordan derivation, block upper triangular matrix algebra.

\section{REFERENCES}

[1] D. BenKović, Jordan derivations and antiderivations on triangular matrices, Linear Algebra Appl. 397 (2005), 235-244.

[2] M. BREŠAR, Jordan derivation on semiprime rings, Proc. Amer. Math. Soc. 104 (1988), 1003-1006.

[3] H. Ghahramani, Characterizing Jordan derivations of matrix rings through zero products, Math. Slovaca, in press.

[4] I. N. HersteIn, Jordan derivations on prime rings, Proc. Amer. Math. Soc. 8 (1957), 1104-1110.

[5] B. E. Johnson, Symmetric amenability and the nonexistence of Lie and Jordan derivations, Math. Proc. Camb. Phil. Soc. 120 (1996), 455-473.

[6] N. JaCobson, C. E. Rickart, Jordan homomorphisms of rings, Trans. Amer. Math. Soc. 69 (3) (1950), 479-502.

[7] J. Li AND F. Y. Lu, Additive Jordan derivations of reflexive algebras, J. Math. Anal. Appl. 329 (2007), $102-111$.

[8] A. M. SinclaiR, Jordan homomorphisms and derivations on semisimple Banach algebras, Proc. Amer. Math. Soc. 24 (1970), 209-214.

[9] J. H. ZHANG, Jordan derivations on nest algebras, Acta Math. Sinica, 41 (1998), 205-212.

[10] J. H. ZHANG AND W. Y. YU, Jordan derivations of triangular algebras, Linear Algebra Appl. 419 (2006), 251-255. 